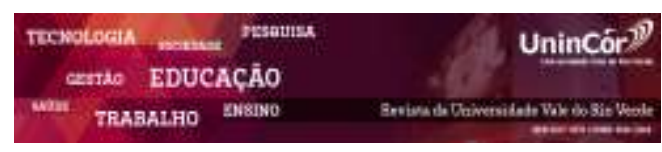

Revista da Universidade Vale do Rio Verde ISSN: 1517-0276 / EISSN: 2236-5362 v. $17 \mid$ n. 1 | Ano 2019

Laís Viera Trevisan Mestranda do Programa de Pós-Graduação em Gestão de Organizações Públicas (PPGOP) da Universidade Federal de Santa Maria (UFSM). Assistente em Administração - UFSM. laisvtrevisan@gmail.com

Shani Carvalho Ceretta Mestranda do Programa de Pós-Graduação em Gestão de Organizações Públicas (PPGOP) da Universidade Federal de Santa Maria (UFSM). Auxiliar em Administração - UFSM. shaniceretta@gmail.com

Gustavo de Souza Carvalho Mestrando do Programa de Pós-Graduação em Gestão de Organizações Públicas (PPGOP) da Universidade Federal de Santa Maria (UFSM).

Técnico em artes gráficas - UFSM. gustavodesouzacarvalho@gmail.com.

Luis Carlos Zucatto Professor Adjunto do Programa de Pós-Graduação em Gestão de Organizações Públicas (PPGOP) da Universidade Federal de Santa Maria (UFSM). luiszucatto@gmail.com

Daniel Arruda Coronel

Professor Adjunto dos Programas de Pós Graduação Gestão de Organizações Públicas (PPGOP), do de Agronegócios e do de Economia e Desenvolvimento (PPGED) da Universidade Federal de Santa Maria (UFSM). Diretor da Editora da UFSM. daniel.coronel@uol.com.br

\section{EXTENSÃO UNIVERSITÁRIA E “THIRD MISSION": UMA ANÁLISE BIBLIOMÉTRICA}

\begin{abstract}
RESUMO
Este estudo trata-se de uma análise bibliométrica a respeito dos temas "extensão universitária" e "third mission". Assim, buscouse identificar as principais características das publicações, as principais instituições, países e áreas de conhecimento que mais publicam sobre as temáticas. Para tal, foi realizada uma pesquisa bibliométrica na base de dados Scopus, no período de 2006 a 2016. Além disso, investigou-se o que está sendo abordado pelas publicações mais citadas nessa base nas temáticas ciências sociais, artes e humanidades, e negócios, gestão e contabilidade. Os resultados apontaram que a área que mais publica acerca de "extensão universitária" e "third mission" são as Ciências Sociais. Ademais, foi possível perceber que esse tema tem maior representatividade no idioma em inglês. Evidenciou-se também que os resultados obtidos para os termos nos idiomas pesquisados possuem abordagens um tanto distintas entre si. Enquanto para "third mission" recorrem temáticas sobre extensão universitária sob um enfoque empreendedor, para o termo em português o enfoque é dado para o SUS.
\end{abstract}

Palavras-chave: Estudo Bibliométrico. Scopus. Extensão Universitária. Third Mission. Ciências Sociais.

\section{UNIVERSITY EXTENSION AND "THIRD MISSION": A BIBLIOMETRIC ANALYSIS}

\begin{abstract}
This study is a bibliometric analysis on the topics "university extension" and "third mission". Thus, we sought to identify the main characteristics of publications, the main institutions, countries and areas of knowledge that publish the most on the issues. To do this, a bibliometric survey was carried out in the Scopus database, from 2006 to 2016. In addition, we investigated what is being approached by the most cited publications on this subject in social sciences, arts and humanities, and business, management and accounting. The results indicated that the area that publishes most about "university extension" and "third mission" is the Social Sciences. In addition, it was possible to perceive that this subject has greater representativeness in the language in English. It was also evidenced that the results obtained for the terms in the languages studied have somewhat different approaches. While for "third mission" they use themes on university extension broadly, under an entrepreneurial approach, for the Portuguese term the focus is given to the SUS.
\end{abstract}

Keywords: Bibliometric Study. Scopus. University Extension. Third Mission. Social Sciences. 


\section{INTRODUÇÃO}

O Brasil tem passado por inúmeras transformações econômicas, sociais, políticas e tecnológicas, o que tem implicado analisar e repensar as inter-relações existentes entre a sociedade, o governo e as instituições.

O Estado atua na sociedade de diversas formas, entre elas, por meio das políticas públicas, que visam assegurar determinados direitos de cidadania. Para Souza (2006), a política pública pode ser explicada como o campo do conhecimento que busca, ao mesmo tempo, colocar o governo em ação e/ou analisar essa ação e, quando necessário, propor mudanças no rumo ou curso desses acontecimentos.

Entre as políticas públicas de um país, inserem-se as universidades públicas, as quais, para Sousa (2010, p. 13), "tem a função de socializar o saber que produz, e desta forma, é também responsabilizada pela integração social dos indivíduos". Nesse cenário, têm-se as práticas de extensão universitária como elo entre academia e sociedade, tratando-se da relação social do saber científico através do contato dos indivíduos da academia com o exterior.

A Constituição de 1988 dispôs o princípio de indissociabilidade entre ensino, pesquisa e extensão. Assim, a universidade passou a inserir a extensão como componente de sistema de aprendizado ofertado pelas instituições, concebendo o conhecido "tripé acadêmico" (FLORIANO et al., 2017). De acordo com Coelho (2014), o Brasil é um país precursor em termos conceituais e práticos no campo da extensão universitária. Contudo, há uma escassez de pesquisas nacionais sobre o impacto da extensão na formação universitária.

Nesse sentido, dois fatos que podem contribuir para incrementar as investigações nesse campo são o aumento da inclusão de jovens nas universidades e um interesse renovado da sociedade nas instituições de ensino superior, na busca de apoio para buscar soluções e inovação. Ademais, novas perspectivas de financiamento, especialmente advindas do Estado, para as atividades extensionistas também já estão promovendo o interesse investigativo em suas consequências de caráter pedagógico, cultural e social.

Com base nesse contexto, justifica-se a relevância do tema e busca-se, por meio deste estudo, conhecer, por meio de um estudo bibliométrico, as principais características das publicações a respeito do tema, as principais instituições, países e áreas de conhecimento que mais publicam sobre o assunto, no período de 2006 a 2016.

\section{REVISÃO BIBLIOGRÁFICA}

Conforme De Paula (2013), em seus primórdios, a extensão universitária assumiu duas vertentes básicas: a primeira, com origem na Inglaterra, difundiu-se pelo continente europeu envolvendo instituições como o Estado, a Igreja e partidos, e buscou legitimação e estabilidade mediante a implantação do Estado do Bem-Estar Social. A segunda vertente, 
protagonizada pelos Estados Unidos, possuiu como objetivo a maior aproximação da universidade com o setor empresarial, no sentido da transferência de tecnologia.

No Brasil, as primeiras atividades de extensão ocorreram no início do século XX, na Universidade Livre de São Paulo, por meio de conferências e semanas abertas ao público e foi a partir do Decreto n. 19.851, de 11 de abril de 1931 (BRASIL, 1931), o qual estabeleceu as bases do sistema universitário brasileiro, que se começou a fazer referência à extensão como instância realizadora de cursos, conferências e outras atividades educativas no espaço acadêmico.

Para Carbonari e Pereira (2007), ao longo da história da extensão no Brasil, cada instituição praticou a atividade como entendia: as universidades públicas priorizaram a pesquisa, e a extensão era como um mero complemento às outras atividades; já as universidades privadas utilizaram a extensão a fim de obter resultados financeiros, por meio da prestação de serviços remunerados. Assim, as atividades extensionistas foram muito criticadas pelo seu caráter paternalista e assistencialista. Além disso, percebe-se que a implementação da extensão ocorreu em função da orientação normativa da política para ensino superior, e não pela responsabilidade da instituição acerca do cumprimento de suas funções sociais.

Em 1987, foi criado o Fórum Nacional de Pró-Reitores de Extensão das Universidades Públicas Brasileiras (ProExt), no qual as discussões buscavam maior precisão do conceito de extensão, além de organizar a extensão como um órgão próprio dentro da instituição, a fim de articular projetos e atividades. Assim, em 2001, foi elaborado o Plano Nacional de Extensão Universitária pelo Fórum de Pró-Reitores de Extensão das Universidades Públicas Brasileiras e pela Secretaria de Educação Superior do Ministério da Educação e do Desporto, no qual a extensão universitária ficou definida como "o processo educativo, cultural e científico que articula o Ensino e a Pesquisa de forma indissociável e viabiliza a relação transformadora entre Universidade e Sociedade" (p. 2).

Internacionalmente, a extensão universitária é denominada "third mission", com tradução para o português de "terceira missão". Para Mollas-Gallartet et al. (2002), as atividades da terceira missão estão relacionadas a geração, uso, aplicação e exploração de conhecimento e outras capacidades universitárias fora do ambiente acadêmico; dessa forma, ela se preocupa com as interações entre universidades e sociedade. Ainda, ao longo da evolução das universidades, a terceira missão atingiu o estágio em que suas contribuições são vistas como importantes e distintivas, merecendo políticas e recursos específicos para garantir seu funcionamento efetivo.

\section{METODOLOGIA}

Este estudo se desenvolveu a fim de identificar as principais características das publicações a respeito das temáticas "third mission" e “extensão universitária”. Para tal, o trabalho classifica-se metodologicamente como pesquisa bibliométrica de natureza descritiva. 
Neste estudo, a base Scopus foi escolhida para o desenvolvimento da pesquisa, tendo em vista sua ampla base de dados, considerada a maior pela Elsevierí, com mais de 60 milhões de registros para periódicos e 130 mil livros.

A partir disso, foram utilizadas como categorias de análise as expressões "third mission" $\mathrm{e}$ "extensão universitária" - entre aspas para garantir o termo por inteiro - que deveriam constar no título, resumo ou palavras-chave dos artigos. Com isso, objetiva-se analisar o termo em inglês - de ampla abrangência internacional - e em português - como é utilizado no Brasil e demais país de língua portuguesa - que compreende 2006 a 2016, excluído 2017 por não estar concluído no momento da elaboração deste estudo.

Nesta configuração, obteve-se o total de 131 artigos, sendo 113 para a expressão "third mission" e 18 para o termo "extensão universitária”. Na sequência, para análise, foram utilizadas as ferramentas de tabulação do Scopus, analyze search results, a qual traz gráficos com as informações organizadas de modo explícito e ordenado.

Ademais, para averiguar as tendências características mais aprofundadas das temáticas, foram selecionados os três artigos mais citados em cada expressão-chave e o artigo brasileiro mais citado para a expressão "third mission", com o objetivo de evidenciar os maiores interesses dos pesquisadores na área. No entanto, para análise dos artigos mais citados, optou-se pela inserção dos filtros referentes às áreas de social sciences, arts and humanities e business, management and accounting para um retorno mais voltado à área de interesse dos pesquisadores. Por fim, fez-se uma análise dos artigos em língua portuguesa e inglesa.

\section{ANÁLISE E DISCUSSÃO DOS RESULTADOS}

Nesta seção, são apresentadas a quantificação e as características das publicações acerca das categorias "third mission" e "extensão universitária". Para tal, foi verificado o número de publicações por ano para a temática, a quantidade por periódico, as instituições de ensino e países mais recorrentes e a área de ampla abrangência. Ainda, serão caracterizadas as publicações mais citadas para cada uma das expressões.

\subsection{THIRD MISSION: QUANTIFICAÇÃO E CARACTERÍSTICAS DAS PUBLICAÇÕES}

A partir da aplicação dos filtros utilizados como parâmetros para a pesquisa, foram encontrados 113 artigos para a expressão "third mission".

No primeiro ano de análise, houve a publicação de apenas 1 artigo; seguida em 2007 por 2; em 2008 por 5 publicações; e em 2009 por 14, momento em que se percebe um salto significativo em relação aos anos anteriores. Nos anos de 2010, 2011 e 2012, houve um decréscimo nas publicações, com 9, 11 e 5 artigos, respectivamente. Já em 2013, retorna o aumento pelo interesse no tema, com 12 publicações, e 2014 com 14. Ainda que no ano de 2015 o interesse tenha diminuído para 12, 2016 apresentou quantidades expressivas para o período, com 27 artigos publicados, o que revela que, embora não se possa assegurar que haja amplo interesse na temática, a mesma parece estar ganhando lentamente representatividade acadêmica. 
A Figura 1 evidencia o número de publicações sobre "third mission" por ano e periódico. Conclui-se que a fonte líder para o tema "third mission" é a revista Science and
Public Policy, com 14 publicações; seguida pelo Journal Of Technology Transfer, com 8 artigos publicados.

Figura 1 - Número de publicações sobre "third mission" por ano e por periódico

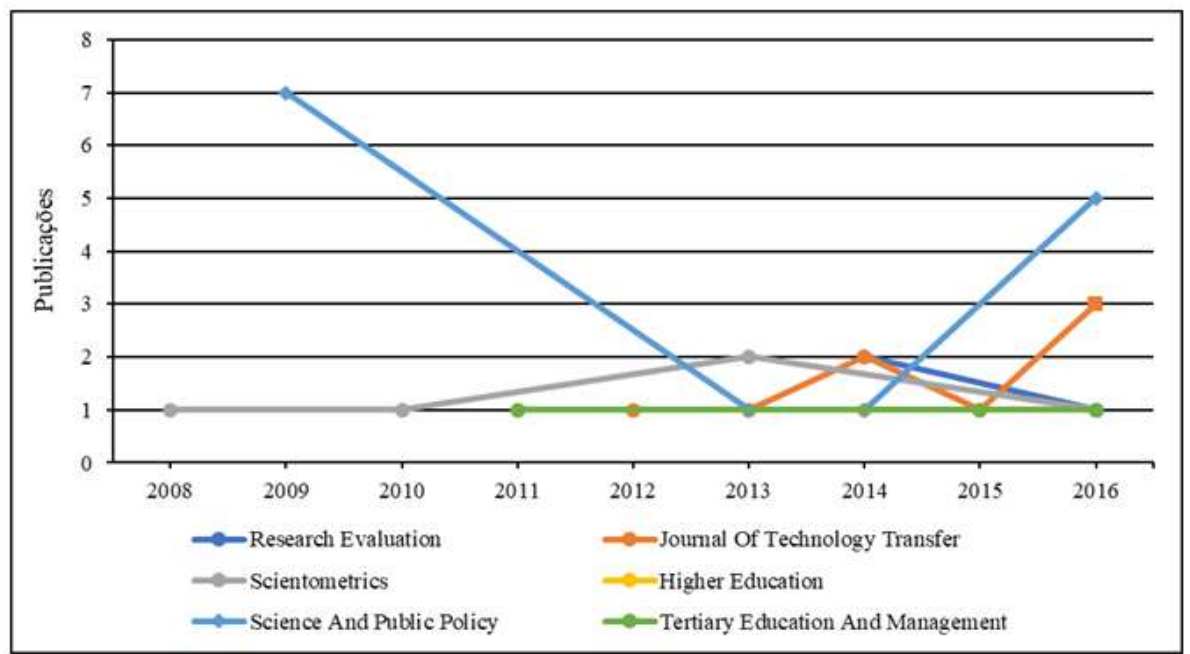

Fonte: adaptada de Scopus (2017).

$\mathrm{Na}$ sequência, realizou-se um comparativo do número de publicações sobre "third mission" de acordo com a instituição de ensino da qual provém o autor. Em primeiro lugar, com 6 publicações, aparece a Universidade de Cambridge. Em segundo lugar, todas elas com 3 publicações por instituição para a temática analisada, a Universidade de Manchester, Universidade Livre de Amsterdã, Universidade de Oslo, Universidade de Pavia, Universidade de Toronto, Universidade Johns Hopkins, Politécnico de Milão, Universidade de Lund e CSIC-UPV Instituto de Gestão de Inovação e Conhecimento do Conselho Nacional de Pesquisa da Espanha e da Universidade Politécnica de Valência.

$\mathrm{Na}$ sequência, identificou-se as publicações sobre "third mission" pelo país de origem. Observou-se que a Itália é o país que mais possui publicações, com 23 artigos sobre a temática. Em segundo lugar, está o Reino Unido, com 19. Os Estados Unidos ocupam a terceira posição, com 16 publicações. O Brasil está em $13^{\circ}$ colocado, com 3 artigos publicados. Esse dado evidencia que o Brasil se apresenta pouco representativo na temática internacionalmente.

$\mathrm{Na}$ Figura 2, são categorizadas as publicações sobre "third mission" por área de conhecimento. Assim, verifica-se que a área do conhecimento que mais possui publicações a respeito do tema "third mission" é a área das Ciências Sociais, representando 52,2\% dos artigos sobre a temática, com 59 publicações. Em segundo lugar, está a área de Administração, com $31 \%$ das publicações, contabilizando 35 artigos. Na terceira posição, está a área de Engenharia, representando $18,6 \%$ das publicações, com 21 artigos. 
Figura 2 - Publicações por áreas do conhecimento

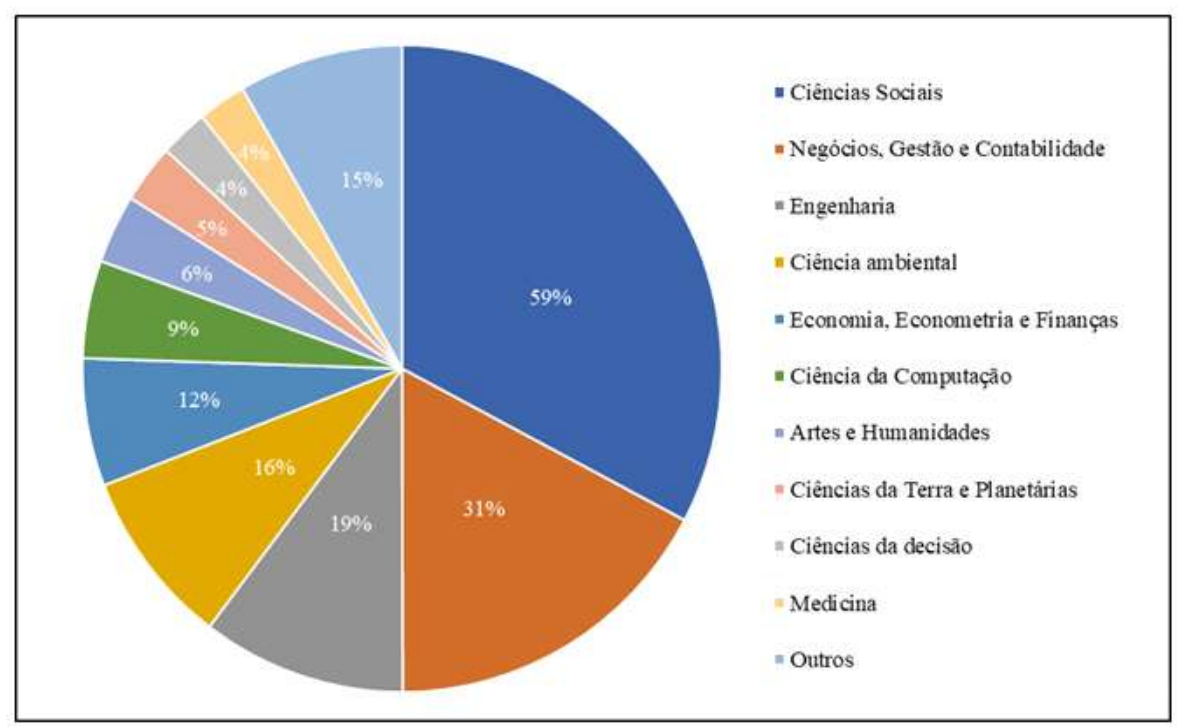

Fonte: adaptada de Scopus (2017).

\subsection{EXTENSÃO UNIVERSITÁRIA: QUANTIFICAÇÃO E CARACTERÍSTICAS DAS PUBLICAÇÕES}

A partir da aplicação dos filtros utilizados como parâmetros para a pesquisa, foram encontradas 18 publicações para "extensão universitária".

Referente ao número de publicações sobre "extensão universitária" por ano, na base pesquisada, observou-se que houve poucas publicações relacionadas à temática "extensão universitária" no período analisado. De 2006 a 2008, não houve artigos publicados; em 2009, houve 3 publicações, passando para 2 em 2010 e 1 em 2011. Em 2012, voltou a não haver nenhuma publicação; já em 2013 passou para 4 publicações, 2 em 2014 e o auge foi atingido em 2015, com 5 publicações, voltando a cair para 1 no ano posterior, 2016. A partir disso, percebe-se que a temática na língua portuguesa possui pouca representatividade acadêmica e amplo potencial de investigação.

A Figura 3 apresenta o número de publicações sobre "extensão universitária" por ano e periódico, revelando que a revista Interface: comunicação, saúde, educação foi responsável pela publicação de 4 artigos, enquanto as demais receberam apenas 1 cada. 
Figura 3 - Número de publicações sobre "extensão universitária” por ano e periódico

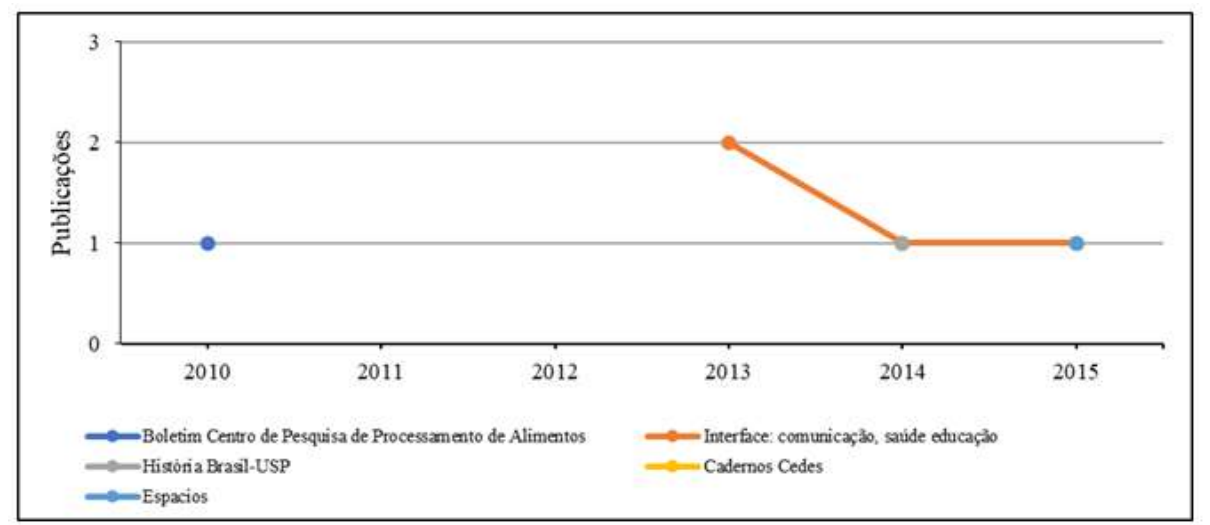

Fonte: adaptada de Scopus (2017).

No que se refere ao número de publicações sobre "extensão universitária”, de acordo com a instituição de ensino da qual provém o autor, em primeiro lugar, com 3 publicações, aparece a Universidade de São Paulo; em segundo lugar, a Universidade Estadual Paulista, Universidade Federal de Santa Catarina, Universidade Federal de Goiás e Universidade Estadual de Maringá, todas elas com 2 publicações por instituição para a temática “extensão universitária”. Das demais instituições (Universidade do Oeste Paulista, Universidade Federal do ABC, Universidade Federal Fluminense, Universidade do Estado do Rio de Janeiro e Universidade Federal da Bahia), cada uma possui apenas um artigo publicado no período. Vê-se, portanto, que, entre as cinco universidades que mais abordaram o tema, duas são paulistas.

Com base nesses dados e apoiando-se no que afirma De Paula (2013) acerca dos primórdios da extensão universitária no Brasil, que teve início em São Paulo em 1911, esse estado possui o tema enraizado há mais tempo e, portanto, maior familiaridade com a ciência e a pesquisa produzida por essas instituições de ensino superior.

A respeito dos países que mais publicam sobre "extensão universitária", observou-se que o Brasil é o país que mais publica, contabilizando 17 artigos. Em segundo lugar está o Reino Unido, com uma publicação, e, ainda, há uma publicação com o país não definido. Esses resultados podem se dever ao fato de que a terminologia "extensão universitária" é utilizada no Brasil.

$\mathrm{Na}$ Figura 4, são categorizadas as publicações sobre "extensão universitária” por área de conhecimento. Assim, verifica-se que a área do conhecimento com mais publicações a respeito da "extensão universitária" são as Ciências Sociais, representando $50 \%$ das publicações, com 9 artigos. Em segundo lugar está a área de Medicina, com 33,3\% das publicações (6 artigos). Na terceira posição está Artes e Humanidades, com 11,1\% das publicações, ou seja, 2 artigos. 


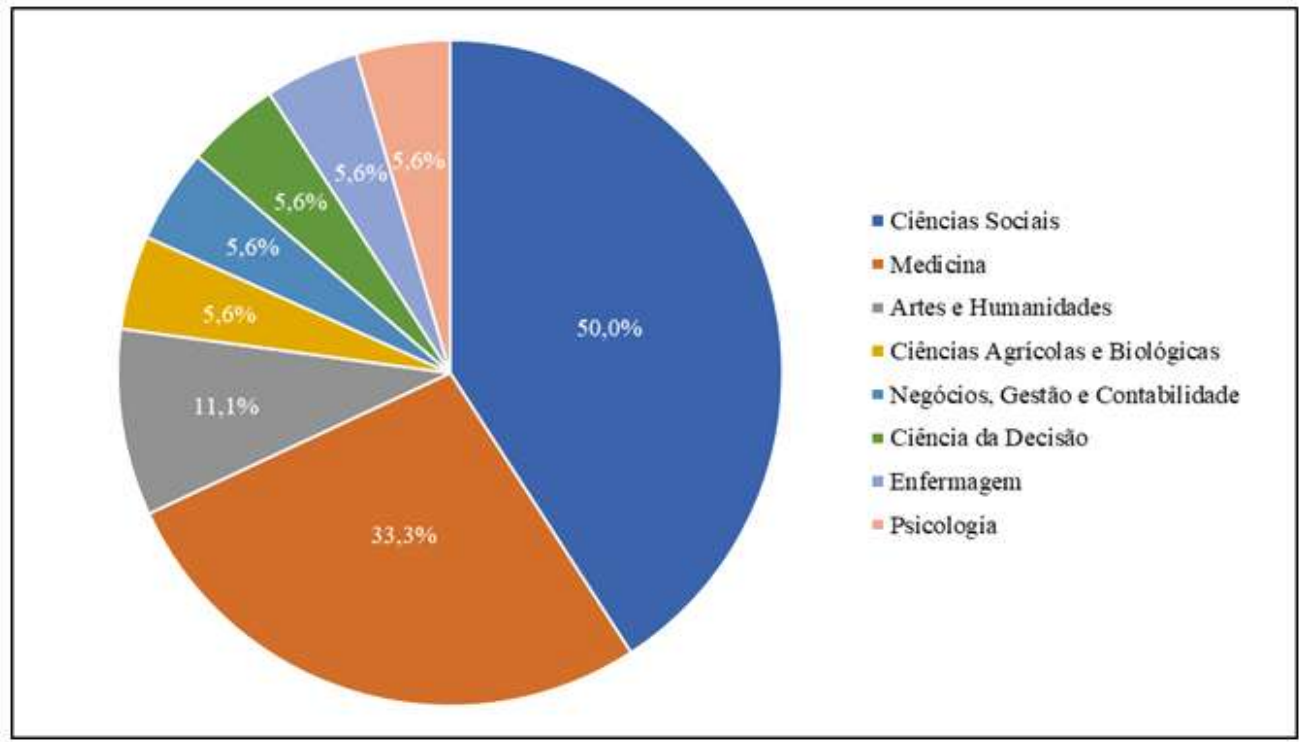

Fonte: adaptada de Scopus (2017).

\subsection{CARACTERIZAÇÃO DAS} PUBLICAÇÕES MAIS CITADAS PARA AS TEMÁTICAS "THIRD MISSION" E "EXTENSÃO UNIVERSITÁRIA"

Compreende-se que é importante analisar as fontes mais citadas pelos artigos de determinada temática, pois estas revelam interesses e tendências da mesma. Para esta análise de conteúdo, utilizaram-se os filtros social Science, arts and humanities e business, management and accounting.

O trabalho de Laredo (2007), intitulado Revisiting the third mission of universities: toward a renewed categorization of university activities? teve 112 citações até 2016 e objetiva uma reflexão a respeito do surgimento da "third mission" nas universidades, como dimensão crítica das atividades desempenhadas, bem como sobre o seu papel para o desenvolvimento a partir da difusão e circulação do conhecimento.

Além deste artigo, também foi analisado um artigo de coautoria de um brasileiro que se revelou a publicação de maior impacto para a temática com origem nacional. $\mathrm{O}$ artigo University start-ups for breaking lock-in soft he Brazilian economy, de autoria de Maculan, A.M. e Carvalho de Mello, J.M., foi publicado em 2009, na revista Science and Public Policy, e possui 10 citações. Esse estudo discorre sobre as três missões das universidades, com maior enfoque para as novas políticas públicas de apoio ao processo de inovação, que foram implementadas visando incentivar as universidades a assumir um papel mais ativo e expandir sua relação com o setor produtivo, comercializando os resultados de suas atividades de pesquisa.

Depois de conhecer estas publicações", verifica-se, agora, o resultado mais citado para a expressão-chave "extensão universitária".

$\mathrm{O}$ artigo de Biscarde, Pereira-Santos e Silva, intitulado Healthcare training, university extension and the National Health System (SUS): necessary connections between knowledge and intervention centeredon reality and 
repercussions with in the education al process foi publicado em 2014 é o mais citado na temática "extensão universitária", com 3 referenciações. O texto descreve a vivência experienciada por graduandos durante o projeto extensionista VIVER SUS Recôncavo, elaborado pela Universidade Federal do Recôncavo da Bahia (UFRB) e desenvolvido no Sistema Único de Saúde (SUS), de forma articulada entre universidade e gestores municipais da região de abrangência da universidade.

\subsection{COMPARAÇÃO DOS RESULTADOS}

Realizando uma comparação das publicações entre os termos "extensão universitária" e "third mission" na base de dados Scopus, foi possível identificar o comportamento das publicações entre os anos de 2006 e 2016, as principais instituições, países e áreas de conhecimento que mais publicam sobre os temas.

No que tange à quantidade de publicações sobre a temática pesquisada, verifica-se que o termo em português possui menor número (18) que em inglês (113). Alguns fatores que podem justificar esse resultado referem-se ao idioma, já que o inglês é considerado uma língua universal; outro pode ser associado aos periódicos voltados à temática que fomentam publicações acerca do tema.

Em relação aos periódicos que mais publicam a respeito da temática, todos possuem seus conteúdos voltados para as políticas públicas. No termo em inglês, as revistas que mais publicam são Science and Public Policy, voltada para políticas públicas de ciência, tecnologia e inovação e Journal of Technology
Transfer, a qual possui enfoque em pesquisas sobre práticas de gestão e estratégias para transferência de tecnologia. No termo em português, a revista que mais publica é a Interface - Comunicação, Saúde e Educação, que possui enfoque na difusão de conhecimento em torno das questões contemporâneas que desafiam o campo da saúde e sua articulação com a comunicação e educação.

A respeito dos países que mais publicam sobre a temática, constatou-se que, na língua portuguesa, o Brasil lidera o ranking e, na língua inglesa, as publicações provêm, em maior número, da Itália, Inglaterra e Estados Unidos, respectivamente. Pode-se levar em consideração que, de acordo com De Paula (2013), esses países são os precursores da temática no mundo e já a têm consolidada, fator que pode justificar tais índices.

Ainda assim, percebe-se que, embora a Itália seja o país com a maior quantidade total de publicações, são a Universidade de Cambridge e a Universidade de Manchester, da Inglaterra, que dominam as duas primeiras colocações. Já no Brasil, é da Universidade de São Paulo que advém a maior quantidade de publicações. Esse resultado corrobora as pesquisas publicadas pelo SIR World Report ${ }^{\mathrm{ii}}$ a respeito da origem das produções científicas mundiais no ano de 2017, o qual informa que, das universidades brasileiras, a Universidade de São Paulo (USP) é a instituição mais produtiva e mundialmente está em $71^{\circ}$ lugar.

Acerca das áreas de conhecimento que mais abordam a extensão universitária, tanto em português quanto em inglês, a área que apresentou maior publicação foi a de Ciências 
Sociais. Esses resultados podem ser explicados em função de que a extensão universitária é uma política pública de Estado e que a área de Ciências Sociais é uma das áreas que mais investigam sobre o tema.

Por fim, analisando as publicações que receberam maior quantidade de citações, segundo a base Scopus, evidencia-se que os resultados obtidos para os termos nos idiomas pesquisados possuem abordagens um tanto distintas entre si. Enquanto para "third mission" recorrem temáticas sobre extensão universitária de modo amplo, sob um viés empreendedor e com enfoque sustentável, para o termo em português o viés dado é para o SUS e para o progresso alcançado pelas atividades de extensão no Brasil.

\section{CONCLUSÕES}

Este trabalho buscou conhecer, por meio de um estudo bibliométrico, as principais características das publicações a respeito de "extensão universitária" e "third mission" em relação às principais instituições, países e áreas de conhecimento que mais publicam sobre o tema.

Pode-se afirmar que, devido à pouca representatividade acadêmica de pesquisas no termo em português, há um amplo potencial de investigação para este campo no Brasil e uma necessidade de internacionalização da ciência brasileira.

Nesse sentido, entre as proposições do estudo, sugere-se a pesquisadores e instituições brasileiros um esforço ainda maior em publicar e desenvolver iniciativas de extensão universitária.

\section{REFERÊNCIAS}

BISCARDE, D. G. S.; PEREIRA-SANTOS, M.; SILVA, L. B. Healthcare training, university extension and the National Health System (SUS): necessary connections between knowledge and intervention centered on reality and repercussions within the educational process. Interface, v. 18, n. 48, p. 177-186, 2014.

BRASIL. Decreto n. 19.851, de 11 de abril de 1931 . Disponível em:

<http://www2.camara.gov.br/legin/fed/decret/19301939/decreto-19851-11-abril-1931505837-

publicacaooriginal-1-pe.html>. Acesso em: 26 out. 2017.

CARBONARI, M. E. E.; PEREIRA, A. C. A extensão universitária no Brasil, do assistencialismo à sustentabilidade. Revista de Educação, Valinhos (SP), v. 10, n. 10, p. 23-28, 2007.

COELHO, G. C. O papel pedagógico da extensão universitária. Em Extensão, Uberlândia, v. 13, n. 2, p. 11-24, jul./dez. 2014.

DE PAULA, J. A. A extensão universitária: história, conceito e propostas. Interfaces, Belo Horizonte, v. 1, n. 1, p. 5-23, jul./nov. 2013.

FLORIANO, M. D. P. Extensão universitária: a percepção de acadêmicos de uma universidade federal do estado do Rio Grande do Sul. Em Extensão, Uberlândia, v. 16, n. 1, p. 9-35, jan./jun. 2017.

LAREDO, P. Revisiting the third mission of universities: toward a renewed categorization of university activities? Higher Education Policy, v. 20, n. 4, p. 441-456, 2007.

SOUSA, A. L. A história da extensão universitária. 2. ed. Campinas: Alínea, 2010.

SOUZA, C. Políticas públicas: uma revisão da literatura. Sociologias [online], n. 16, p. 20-45, jul./dez. 2006.

\footnotetext{
' Disponível em:

<https://www.elsevier.com/americalatina/ptbr/scopus >. Acesso em: 09 out. 2017.

ii Disponível em:

<http://www.scimagoir.com/rankings.php?country=BR A\&year=2011>. Acesso em: 09 out. 2017.
} 